\title{
Simulations of dual morphology in spiral galaxies
}

\author{
S. L. Berman ${ }^{\star}$ \\ Theoretical Physics, University of Oxford, 1 Keble Road, Oxford, UK \\ Received 17 February 2003 / Accepted 15 September 2003

\begin{abstract}
Gas and stars in spiral galaxies are modelled with a combination of hydrodynamic and $N$-body techniques. The simulations reveal morphological differences mirroring the dual morphologies seen in $B$ and $K^{\prime}$ band observations of many spiral galaxies: gaseous images have tighter pitch angles, are more asymmetric, more flocculent and more likely to have multiple arms. Morphological decoupling increases as the stellar arm-interarm contrast and the $Q$ parameter fall. The flocculence of a galaxy is quantified by decomposing the images into logarithmic spirals and defining a parameter closely related to the uniformity of the resulting 2D Fourier spectrum. Thus, a significant amount of morphological decoupling in spiral galaxies is shown to be due to the difference in the dynamics of stars and gas, rather than dust, star formation or galaxy interactions.
\end{abstract}

Key words. hydrodynamics - galaxies: ISM - galaxies: structure - galaxies: spiral - galaxies: fundamental parameters infrared: galaxies

\section{Introduction}

Despite the fact that young and old stars orbit in the same potential, the morphology of infrared and optical images can be uncorrelated (Block \& Puerari 1999; Grosbol \& Patsis 1998; Eskridge et al. 2000). In this paper, DUAL, a combined hydrodynamic and $N$-body code capable of modelling the 2D dynamics of gas and stars in a spiral galaxy, is used to try to simulate the observations of the dual morphologies seen in optical and infrared images, and to discover whether predictions can be made about the intrinsic characteristics of spiral galaxies from their morphologies.

Section 2 briefly describes the theoretical motivation for this project. In Sects. 3 and 4, details are provided of the models and the DUAL code used to recreate dual morphology galaxies and the various methods used to analyze them. The results are presented in Sect. 5, and discussed further in Sect. 6. Conclusions and suggestions for future work are presented in Sect. 7.

\section{Theoretical considerations}

Spiral galaxy disks can be decomposed into two dynamically distinct components. One forms only a small part $(\sim 5 \%)$ of the mass of a galaxy disk and is composed of objects that evolve fast: young hot $\mathrm{O}$ and $\mathrm{B}$ stars, cold interstellar gas, and dust. Dynamically this part of the disk is "cool", being characterized by small random motions of $\sim 10 \mathrm{~km} \mathrm{~s}^{-1}$ thus forming a thin disk.

\footnotetext{
* e-mail:simon@thphys.ox.ac.uk
}

The second component forms the bulk of the mass of the galaxy and is predominantly composed of old $\mathrm{K}$ and $\mathrm{M}$ dwarf stars (Binney \& Merrifield 1998). It is dynamically “warm” and hence stable, and characterized by a vertical velocity dispersion of $\sim 20 \mathrm{~km} \mathrm{~s}^{-1}$ (Dehnen \& Binney 1998) and a thicker disk.

In order to model dual morphology galaxies using $N$-body and hydrodynamic techniques, it is assumed that the light in $K^{\prime}$ band images faithfully traces the underlying mass distribution of the galaxy being observed and that $B$ band observations do not.

Rhoads (1998) deduce that young red supergiants contribute only $\sim 3 \%$ to the global $K^{\prime}$ band flux but upto $33 \%$ in local features. And Rix \& Zaritsky (1995) cite an upper limit of $20 \%$ for the contamination of $K^{\prime}$ band luminosity by young stars. Hence the $K^{\prime}$ band is generally a good tracer of the underlying mass distribution but a possible bias exists in arminterarm contrast amplitudes and other measurements of galactic substructure.

Rix \& Zaritsky (1995) also show that $30 \%$ of the luminosity emitted by stars in $B$ band ( $445 \mathrm{~nm}$ ) images comes from a young stellar population (less than a few dynamical periods old), and $35 \%$ from an old population (older than tens of dynamical periods). However, old stars are more evenly spread around a galaxy disk, hence their contribution to $B$ band luminosity is global rather than local. But young stars, and the gas from which they are formed, are concentrated in star forming regions, and, in the absence of strong shear, these will also be regions of high gas density. Interstellar gas can thus be modelled using isothermal hydrodynamics.

The existence of regular one or two armed spiral structures in the infrared and the multi armed nature of optical spirals, can 
be partly accounted for within the modal theory of spiral structure of Bertin \& Lin (1996). This theory asserts that large scale spiral structure is long lasting and determined by intrinsic properties of galaxies (warm or cool stellar disks, gas mass fraction, etc.) rather than by external effects (mergers, collisions, etc.).

\section{Modelling dual morphology galaxies}

To model observations of dual morphology galaxies, the gas is represented by an isothermal fluid, the stars by $N$-body particles and the background dark halo by an analytic potential. The stars and gas both feel the potential of the dark halo and the stars. The gas is not self gravitating, so the stars do not feel the gas.

The units used in this paper are the disk scale length $R_{\mathrm{d}}$ and the disk mass $M_{\mathrm{d}}$ with $G$ set equal to unity. Thus the unit of time is $t_{\mathrm{d}}=\left(R_{\mathrm{d}}^{3} / G M_{\mathrm{d}}\right)^{1 / 2}$. Although all results are presented in these units, it is sometimes helpful to transform to kpc, $M_{\odot}$ and Gyrs. In such cases, the canonical values of $R_{\mathrm{d}}=5 \mathrm{kpc}$ and $M_{\mathrm{d}}=2 \times 10^{11} M_{\odot}$ are used, which gives a dynamical time of $t_{\mathrm{d}} \sim 10^{7} \mathrm{yrs}$ and a velocity of $v_{\mathrm{d}} \sim 420 \mathrm{~km} \mathrm{~s}^{-1}$.

The grid on which both the hydrodynamic and $N$-body calculations take place is two dimensional and Cartesian, and the shortest distance from the centre to the edge $R_{\mathrm{G}}=7.5 \mathrm{disk}$ scale lengths.

\subsection{The stellar disk}

The stars form a one component disk with exponential surface density and radial velocity dispersion profiles,

$\Sigma_{\mathrm{d}}(R)=\Sigma_{\mathrm{d}} \exp \left(-R / R_{\mathrm{d}}\right)$,

$\sigma_{\mathrm{R}}(R)=\sigma_{\mathrm{R}} \exp \left(-R / R_{\sigma}\right)$,

where $R_{\mathrm{d}}=1$ and $\Sigma_{\mathrm{d}}=0.5 / \pi$ to ensure that $M_{\mathrm{d}}=2 \pi \Sigma_{\mathrm{d}} R_{\mathrm{d}}^{2}=1$. For an exponential disk, the maximum circular speed is 0.62 which occurs at $R=2.15$.

Although simulations all take place in 2D the stellar particles have an effective scale height due to their velocity dispersion, the softened gravitational forces which are used in $N$-body simulations and the effect of the interpolation of gravitational forces from the grid to the positions of the particles. Following van der Kruit \& Searle (1981), I assume the stellar disk to be a locally isothermal sheet, which implies that

$\rho(z)=\rho_{0} \operatorname{sech}^{2}\left(z / z_{0}\right)$,

$z_{0}=\sigma_{z}^{2} / \pi G \Sigma_{\mathrm{d}}$,

where $\sigma_{z} \sim 0.6 \sigma_{\mathrm{R}}$ (Dehnen \& Binney 1998) is the vertical velocity dispersion. Adopting $R_{\sigma}=2$ gives a constant scale height of $z_{0}=0.72 \sigma_{\mathrm{R}}^{2}$, where $\sigma_{\mathrm{R}}$ remains to be determined. This is consistent with the scale height of late type galaxies being approximately constant (de Grijs \& Peletier 1997).

This scale height is equated to $s_{\text {grid }}$, the effective height of the 2D $N$-body particle distribution. Restricting the $N$-body softening length to $0.5<s<2.0$ grid cells (Sellwood, private communication) gives an effective height of $1.2<$ $s_{\text {grid }}<2.3$ grid cells, and restricting the scale height to $0.10<$ $z_{0}<0.14$ (van der Kruit \& Searle 1981) limits the number of grid cells to $250 \times 250$. Choosing $N=2 \times 10^{5}$ particles to model the stellar component gives a mean of 3 particles per grid cell.

\subsection{The dark halo}

The form of the dark halo potential is an isochrone, used because it has a core rather than a cusp to its density profile, falls to zero density at large radii and its potential and circular speed can be defined using just the halo mass $M_{\mathrm{h}}$ and its scale length $R_{\mathrm{h}}$. The slope of the total rotation curve at four disk scale lengths is fixed at zero. Since the disk is already determined, the halo mass and scale length are both fixed by choosing the disk fraction at four disk scale lengths $f_{\mathrm{d}}$.

When $f_{\mathrm{d}} \geq 0.4$ (true for 9 out of 16 models) the dark matter content within one disk scale length is minimal $(<15 \%)$ and hence the galaxy model is dominated by the disk. At the lower value of $f_{\mathrm{d}}=0.3$, the halo mass fraction at one disk scale length rises to $27 \%$.

\subsection{Stellar distribution function}

The stellar particles' initial conditions are drawn from the warm disk distribution function of Dehnen (1999),

$f(E, L)=\frac{\gamma\left(R_{E}\right) \Sigma_{\mathrm{d}}\left(R_{E}\right)}{2 \pi \sigma_{\mathrm{R}}^{2}\left(R_{E}\right)} \exp \left[\frac{\Omega\left(R_{E}\right)\left[L-L_{\mathrm{c}}(E)\right]}{\sigma_{\mathrm{R}}^{2}\left(R_{E}\right)}\right]$,

where $R_{E}$ and $L_{\mathrm{c}}(E)$ are the radius and angular momentum of the circular orbit at energy $E, \Omega$ is the angular velocity and $\gamma \equiv 2 \Omega / \kappa$. Particles are chosen from this distribution function within radii smaller than $R_{\text {cut }}=5$ to ensure that particles stay within the grid. Since an exponential disk extends to infinity, the mass of the stellar disk on the grid is only 0.96 mass units. The distribution function $f(E, L)$ is sampled using the procedure and accompanying code of Dehnen (1999) to generate initial positions and velocities of the stellar particles with minimal noise.

\subsection{Stability and rotation}

The stability parameter $Q$ (Toomre 1964) determines the response of the stellar disk to axisymmetric instabilities. For the disk and halo defined above, defining $Q(R)$ fixes $\sigma_{\mathrm{R}}(R)$. In fact, choosing the minimum value of $Q(R), Q_{\min }$, will fix $\sigma_{\mathrm{R}}$. The left hand panel of Fig. 1 shows the radial profile of $Q$ at the start of a typical simulation.

Figure 2 shows the total rotation curve of the model and the contribution of the stellar and dark components for a typical simulation. The model (dashed) is in reasonable agreement with observed rotation curves of low surface brightness (dotted) and high surface brightness (solid) late type galaxies (normalized to their rotation velocity at two scale lengths) from Swaters et al. (2000). The right hand panel of Fig. 1 shows the angular velocity profile derived from this rotation curve. From this and the determination of the bar pattern speed from the simulation results (see Sect. 4.8), it can be seen that these models have no Inner Lindblad Resonance. 

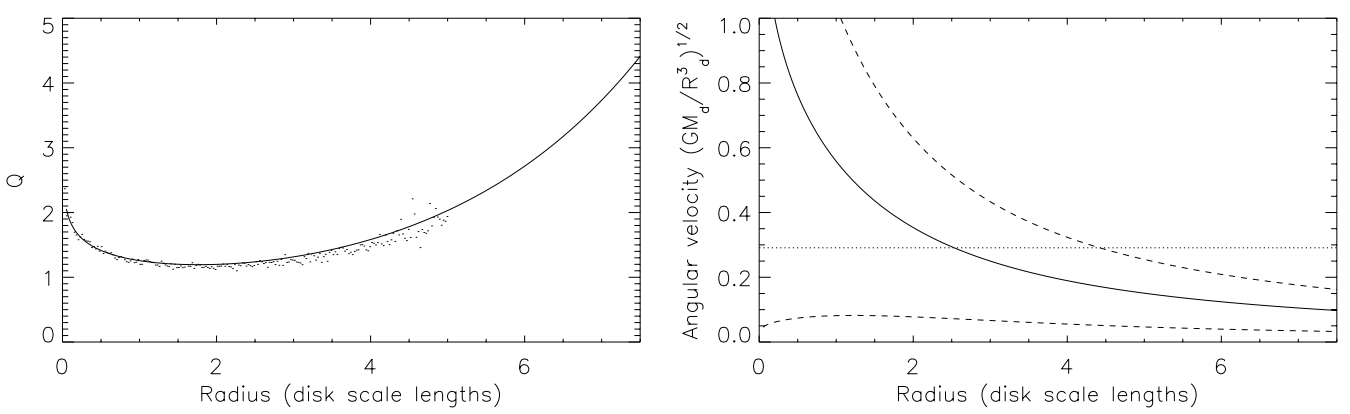

Fig. 1. Left panel: radial profile of $Q$ at the start of sta19. Dots show $Q$ values derived from initial particle positions. The solid line is the theoretical profile based on an exponential disk and isochrone halo. Right panel: angular velocity profiles for gas 19 . The solid line is $\Omega(R)$, the dashed lines are $\Omega(R) \pm \kappa(R) / 2$ and the dotted line is the bar pattern speed $\Omega_{\mathrm{p}}$ which is determined from the results of the simulations.

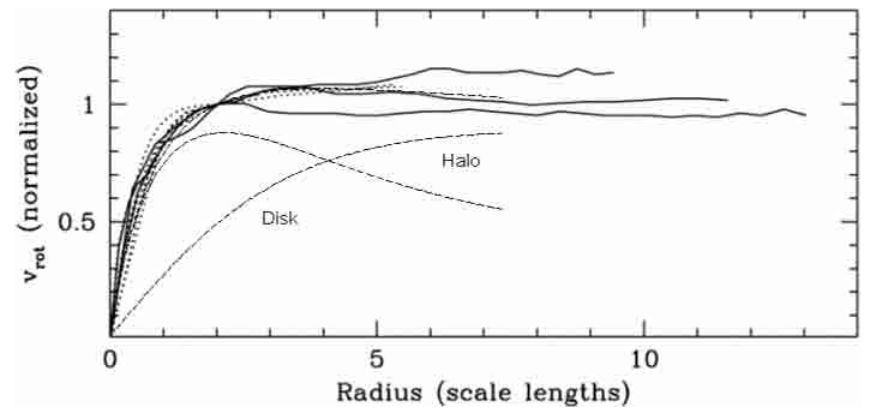

Fig. 2. Rotation curve for a typical simulation showing the total model and the contribution from the stellar disk and dark halo (dashed lines). Also shown are observed rotation curves from Swaters et al. (2000) for late type HSB and LSB galaxies, normalized to their rotation velocity at two scale lengths.

\subsection{The gaseous disk}

The response of the gas is governed by the isothermal Euler equations with gravitational source terms as implemented by the FS2 algorithm (van Albada \& Roberts 1981; van Albada et al. 1982). In these models there is no fixed pattern speed i.e. simulations take place on the inertial frame. The sound speed is set to 0.025 , equal to $10 \mathrm{~km} \mathrm{~s}^{-1}$ assuming the canonical values of length and mass given in Sect. 3. The gas is initially set on circular orbits at a uniform surface density $\Sigma_{\mathrm{g}}$.

Englmaier \& Gerhard (1997) find significant differences in gas shock morphology when the sound speed is changed in their simulations. To discover what effect the sound speed has on these simulations, I ran variations of models with grand design (run 19) and multi armed (run 16) gas spirals using sounds speeds of 0.01 and 0.05 . In both models, the smallest scale structures are wiped out as the sound speed rises. Higher sound speeds also lead to lower density contrasts in the gas spirals. However, the values of pitch angle and flocculence (as defined in Sect. 4) are unchanged. Hence at sound speeds below 0.05 , the conclusions of this study remain valid. Above this, the reduction in small scale structure would bring the stellar and gaseous morphologies closer to one another.

\subsection{The DUAL code}

DUAL is a combination of the finite-difference hydrodynamic code GALAHAD introduced in Berman (2001) and a particlemesh $N$-body code written by J. Sellwood. The hydrodynamics solve the isothermal Euler equations with gravitational source terms, neglecting self-gravity, by use of the FS2 algorithm. The $N$-body calculation solves the Poisson equation and Newton's second law to give the motion and gravitational potential of the stellar particles. Both parts of the code operate on a 2D Cartesian grid. The DUAL code provides a powerful and publicly available ${ }^{1}$ tool to investigate many aspects of galaxy dynamics.

\subsection{Simulation run time}

Simulations ran for 160 time units. This can be compared to the rotation time for a particle at $R=2, t_{\text {rot }}(2)=2 \pi R / v_{\mathrm{c}}(2)=$ $12.6 / v_{\mathrm{c}}(2)$, where $v_{\mathrm{c}}(2)$ is the rotation velocity at $R=2$ as determined from the rotation curve. For most simulations $t_{\text {rot }}(2) \sim$ 16-20 and so the simulations run for roughly 8-10 rotation periods.

\section{Analysis techniques}

\subsection{Animating the results}

To view the simulations, I construct animations consisting of 80 time slices taken at intervals of 2 time units. IRAF is used to convert the 3D ASCII data files into FITS format, which are then animated using AIPSVIEW ${ }^{2}$. The animations are used to determine the largest set of contiguous time slices exhibiting two armed spirality within each simulation. Many of the mophological characteristics defined below are calculated for each time slice and the mean value over the set of time slices gives the "time average" for the simulation as a whole.

\footnotetext{
1 The DUAL code can be downloaded from www-thphys.physics.ox.ac.uk/users/SimonBerman

2 available to download at monet.astro.uiuc.edu/aipsview/ av.html
} 


\subsection{Harmonic analysis}

To highlight the underlying spiral structure in a galaxy, the images are decomposed into logarithmic spirals

$R=R(\theta)=R_{0} \exp (\theta \tan \psi)$,

where $\psi$ is the pitch angle, the angle between the normal to the radius vector and the ridge of the spiral-arm, and $R_{0}$ is a radius at which the spiral passes through $\theta=0$.

Danver (1942) and Kennicutt (1981) show that logarithmic spirals can represent real spiral-arms. Hence, much of the structure in a spiral galaxy image can reconstructed using a small number of components of a 2D Fourier transform with logarithmic spirals as basis functions. For a galaxy image transformed onto a regular grid in a $(\ln R, \theta)$ coordinate system, the complex amplitude of each Fourier component is

$A(m, \alpha)=\frac{\sum_{k} I_{k} \exp \left[-\mathrm{i}\left(m \theta_{k}+\alpha \ln R_{k}\right)\right]}{\sum_{k} I_{k}}$,

where $I_{k}(\ln R, \theta)$ is the intensity of the $k$ th pixel. The azimuthal wavenumber or number of spiral-arms $m$ and the logarithmic radial wavenumber $\alpha$ are related to the pitch angle by $\tan \psi=$ $-m / \alpha$.

To perform a Fourier decomposition in $(\ln R, \theta)$ space a minimum and maximum radius, $R_{\min }$ and $R_{\max }$, must be defined. These radii need to be chosen with care because they can have a significant effect on the resulting Fourier spectra. Reducing the minimum radius will increase the value of the calculated pitch angle, particularly when a bar is present. $R_{\max }$ is fixed at 6 scale lengths. $R_{\min }$ is chosen to exclude the bar from the calculations whilst including as much of the data set as possible. Since the Fourier component $P_{2,0}$ picks out the bar from a galaxy image, the radius at which the power of the $P_{2,0}$ component first drops below 0.005 is used as $R_{\min }$. The value of 0.005 is an arbitrary yet consistent one which produces sensible results roughly in line with "by eye" expectations.

The Fourier amplitudes are converted into a normalized power spectrum for the non-axisymmetric part $P_{m \neq 0, \alpha}$. Since it is unlikely that a single Fourier component will reproduce all of the spiral structure in a galaxy, even excluding any central bar, the pitch angle of a particular spiral pattern is given as the pitch angle of the Fourier component with the largest power.

Fourier amplitudes are calculated using 2DFFT (Saraiva Schroeder et al. 1994), a code which utilizes a 2D discrete Fourier transform in which the amplitudes are produced for $-50 \leq \alpha \leq 50$ and $0 \leq m \leq 6$ where $\Delta \alpha=0.25$ and $\Delta m=1$.

\subsubsection{Example power spectra}

Figure 3 shows images and spectra of a grand design spiral in which both the stars and the gas follow a regular two armed structure. The spectra corroborate this since the stars and the gas are strongly peaked in the $m=2$ component. The gas also possesses significant power in the $m=4$ and 6 components, making its arms narrower.

The stellar image of Fig. 4 is also strongly two armed, and this is verified by the corresponding spectra. Although the gaseous image may seem to be a completely flocculent mess, the spectra show that there is a strong $m=4$ structure present with a similar, but smaller, pitch angle to that of the stars. There is also a lot of other structure in the gas image that is not seen in stars.

Lastly, the stellar structure of Fig. 5 is two armed, as can be seen from its spectra. However, the gaseous image is now completely flocculent and its spectrum is strongly peaked at all values of $m$. The many interfering spiral waves prevent any single spiral dominating the image.

All of the gaseous images exhibit ring structures near to and outside of $R_{\max }$ due to boundary effects. Rerunning gas 19 with the gas initially in an exponential disk, removes almost all of the ring structures but leaves the rest of the gaseous structure unchanged.

\subsection{Flocculence}

Grand design spirals have one strong peak in their power spectrum whereas flocculent spirals have multiple peaks. Hence, flocculent power spectra are more evenly distributed than grand design power spectra. The best measure of the uniformity of a distribution is the entropy, $S=-\sum_{m, \alpha} P_{m, \alpha} \ln P_{m, \alpha}$ (Jaynes 1958).

This can be used to characterize the flocculence of an image, but a couple of issues must be considered first:

1. If a galaxy image consists solely of a strong two armed spiral, there will be large contributions from the $m=2 \mathrm{spec}-$ trum. However, the $m=4$ and 6 spectra may also be strongly peaked, creating a narrower spiral-arm, but one which would not be flocculent despite a multiple peaked spectra. Hence the $m=4$ and 6 spectra are ignored in calculating the flocculence.

2. Almost all galaxies have many weak components in their power spectra - the noise. These components flattens the distribution and tends to blur the distinction between grand design and flocculent images. Hence only components with power $P_{m, \alpha}>0.25 \max \left(P_{m, \alpha}\right)$ are considered.

After these considerations, there are an average of 123 components remaining. These are renormalized before calculating the entropy to ensure that $\sum_{m, \alpha} P_{m, \alpha}=1$.

The entropy $S$ lies in the range $0 \leq S \leq \ln N_{\max }$, where $N_{\max }=1604$ is the number of non zero $P_{m, \alpha}$ contributing to $S$. We want flocculence $\mathcal{F}$ to be a linear function of $S$ defined so that $0 \geq \mathcal{F} \geq 1$. We could choose $\mathcal{F}=S / \ln N_{\max }$ but this has the drawback that $\mathcal{F}$ depends on $N_{\max }$. This is avoided by defining

$\mathcal{F}=\frac{S-\ln \left(N_{\max } / 1000\right)}{\ln 1000}$

The maximum entropy would arise from a completely flat distribution. Similarly, the entropy of each image can be associated with a Fourier spectrum in which a number of the components $n$ are equal in power and the rest have zero power. Since the form of the flocculence function is logarithmic rather than linear, an arithmetic increase in $\mathcal{F}$ leads to a geometric 

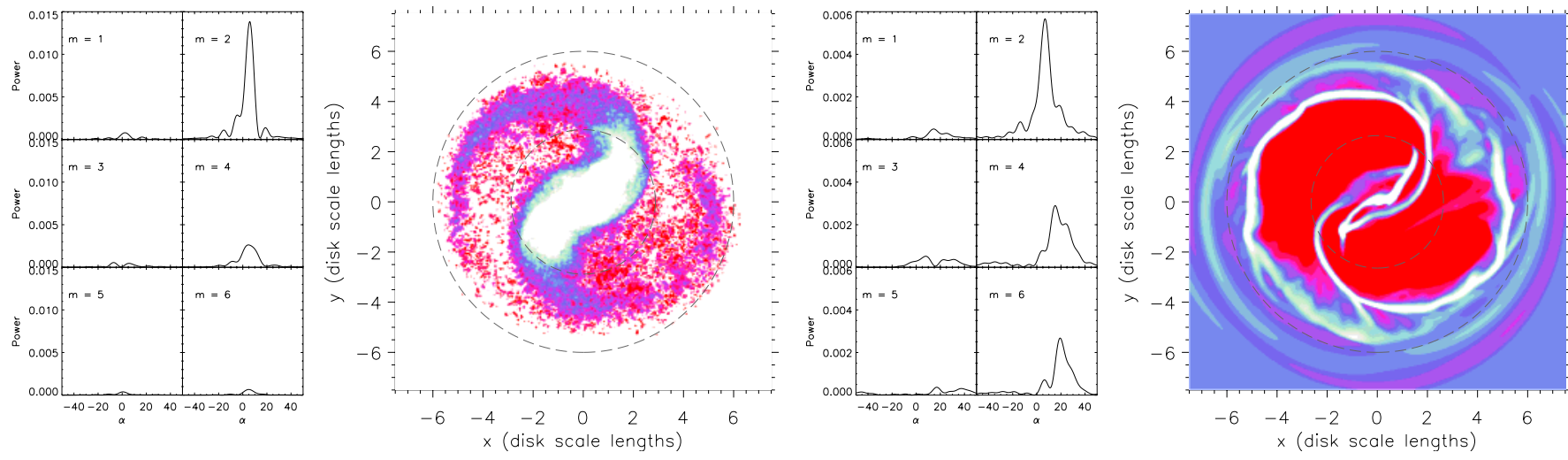

Fig. 3. Stellar and gaseous density fields and harmonic spectra for the grand design spiral sta19 and gas 19 . The dashed lines in the galaxy images indicate the minimum and maximum radii used in the Fourier analysis. The flocculence, asymmetry and two armed pitch angles are $\mathcal{F}=0.51, \mathcal{A}=0.10$ and $\psi=20.9^{\circ}$ for the stars and $\mathcal{F}=0.53, \mathcal{A}=0.13$ and $\psi=16.5^{\circ}$ for the gas. The stellar arm-interarm contrast is $C=2.48$.
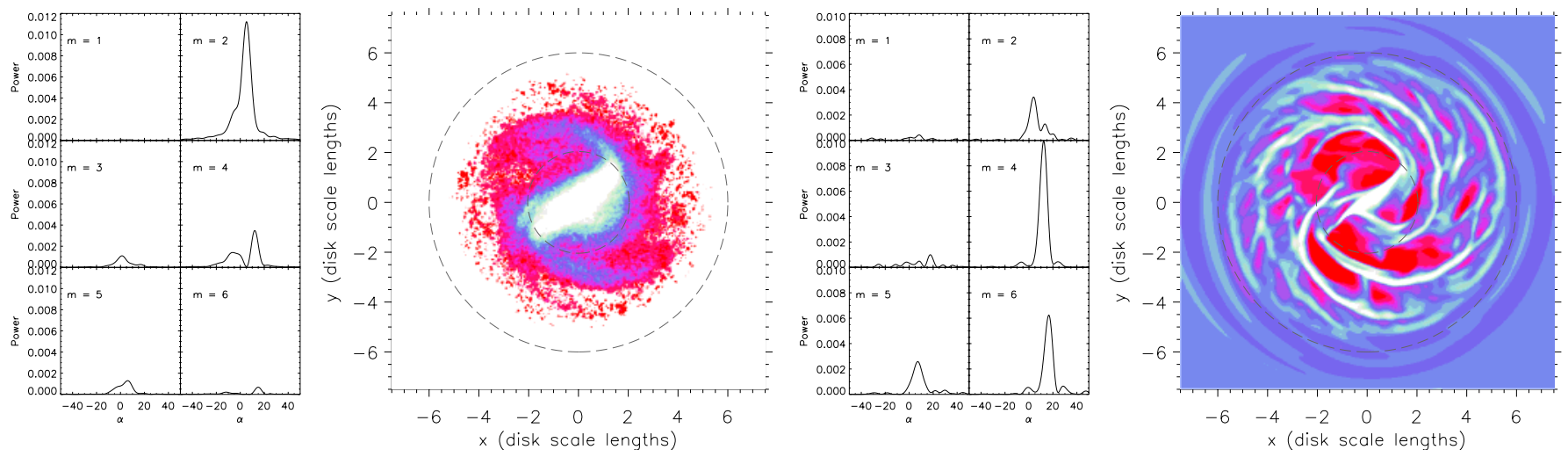

Fig. 4. Stellar and gaseous density fields and harmonic spectra for the multi armed spiral sta16 and gas 16 . The dashed lines in the galaxy image indicate the minimum and maximum radii used in the Fourier analysis. The flocculence, asymmetry and pitch angles are $\mathcal{F}=0.53$, $\mathcal{A}=0.13$ and $\psi=20.9^{\circ}$ (two arms) for the stars and $\mathcal{F}=0.61, \mathcal{A}=0.22$ and $\psi=17.7^{\circ}$ (four arms). The stellar arm-interarm contrast is $C=1.71$.
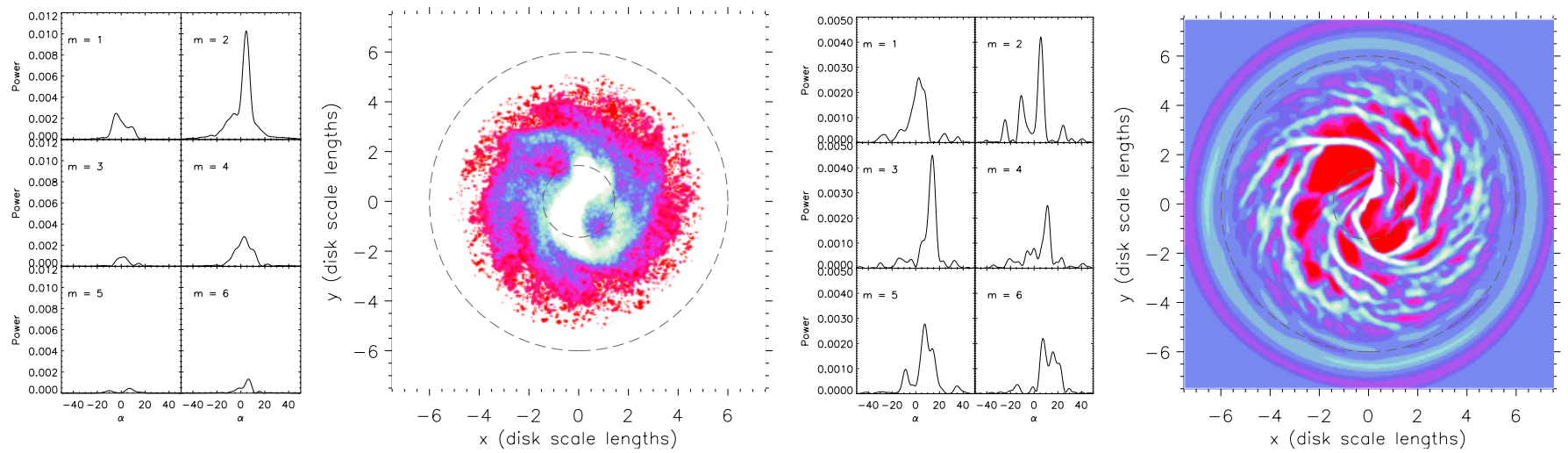

Fig. 5. Stellar and gaseous density fields and harmonic spectra for the flocculent spiral sta44 and gas 44 . The dashed lines in the galaxy image indicate the minimum and maximum radii used in the Fourier analysis. The flocculence, asymmetry and pitch angles are $\mathcal{F}=0.47, \mathcal{A}=0.23$ and $\psi=24.0^{\circ}$ (two arms) for the stars and $\mathcal{F}=0.70$ and $\mathcal{A}=0.54$ for the gas. The arm-interarm contrast is $C=1.58$.

increase in $n$, with $\mathcal{F}=1$ implying that $n=N_{\text {max }}$ and $\mathcal{F}>0$ if $n>N_{\max } / 1000$. For 1000 components, $n$ doubles when $\mathcal{F}$ increases by 0.1 . In this sense, an image is twice as flocculent as another if its flocculence $\mathcal{F}$ is higher by 0.1 .
The above definition produces values of $\mathcal{F}$ which are roughly in line with "by eye" expectations for the simulation images and for test cases of $\mathcal{F}=0.41$ for a grand design spiral, $\mathcal{F}=0.74$ for a flocculent galaxy and $\mathcal{F}=0.80$ for an image composed of pure noise. 
Elmegreen \& Elmegreen (1982) define a classification system for the regularity of spiral-arm structure. Flocculent galaxies have arm classes of 1 to 4 and "patchy", "ragged", "fragmented" or "fleece-like" spiral arms. Using this definition, gas 44 would certainly be called flocculent. However, many other images with medium to high values of $\mathcal{F}$ would probably be classified as arm class 5 or 6: two symmetric inner arms and irregular outer arms.

\subsection{Asymmetry}

The asymmetry $\mathcal{A}$ of an image in the azimuthal direction is defined by Iye et al. (1982) to be the fraction of power in odd- $m$ components. For the grand design test case $\mathcal{A}=0.06$ whereas for the flocculent test image $\mathcal{A}=0.49$. Hence, the asymmetry of an image may also be an indication of its flocculence. Asymmetries are calculated for all simulations and they fall roughly within this range.

\subsection{Radial Fourier analysis}

The disadvantage of decomposing an image into logarithmic spirals is that no radial information survives. To derive radial variations in morphological characteristics, the data are transformed and interpolated from $250 \times 250(x, y)$ coordinates to $256 \times 128(R, \theta)$ coordinates and one dimensional Fourier transforms are performed on the data at each radius. The result is the radial variation of the amplitude and phase of the $m=1 \rightarrow 6$ Fourier components. This information is used in Sect. 4.6 to calculate the arm-interarm contrast and was used in Sect. 4.2 to calculate the minimum radius.

\subsection{Arm-interarm contrast}

The contrast between the spiral-arms and the space in between the arms is not easy to define because it is difficult to determine where the centre of the arm is and what to take as the interarm region. However, it is worth persevering since high contrast stellar spirals are good candidates for producing grand design gaseous spirals whereas low contrast stellar spirals often result in flocculent gaseous spirals. Following Rix (1993), the simulation images are subjected to radial Fourier analysis and the arm-interarm contrast $C$ is defined as the ratio of the maximum to the minimum of the even $m$ components,

$I_{R}(\theta)=\left|a_{0, R}\right|+\sum_{m=2,4,6,8}\left|a_{m, R}\right| \cos \left(m \theta+\phi_{m, R}\right)$,

where $a_{m, R}$ is the $m$ th Fourier component at radius $R$ and $\phi_{m, R}$ is its phase. At each radius,

$C_{R}=\max \left(I_{R}(\theta)\right) / \min \left(I_{R}(\theta)\right)$,

and $C$ is the mean value between $R_{\min }$ and $R=5$. The resulting values are generally between 1.5 and 3.0.

\subsection{Monitoring $Q(t)$}

Although $Q_{\min }$ is one of the input parameters to the models, the way in which $Q$ varies with time is not known at the outset of

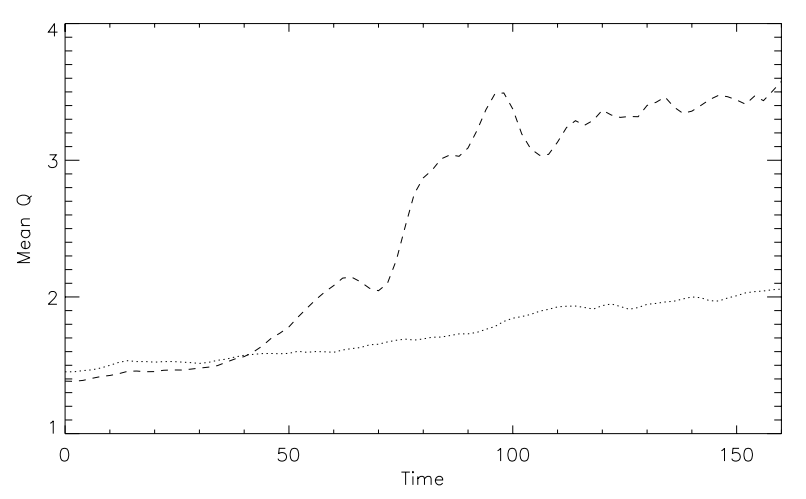

Fig. 6. Evolution of $Q(t)$. The long dashed line shows run sta19 which begins with a low $Q$ value but ends up high. The short dashed line shows sta 16 which starts higher but rises only gradually.

a simulation. It is important to understand the evolution of $Q(t)$ as it is intimately linked to the formation and maintenance of spiral structure. It might be assumed that the value of $Q_{\min }$ at the start of the simulation correlates positively with the mean value of $Q$ at the end of the run. Not so. Figure 6 shows $Q(t)$ for two simulations and is illustrative of the situation in all of the simulations: higher values of $Q_{\min }$ lead to $Q(t)$ which rises slowly whereas lower values of $Q_{\min }$ lead to a rapidly rising and fluctuating $Q(t)$.

\subsection{Pattern speed and resonances}

As mentioned in Sect. 4.2, the Fourier component $P_{2,0}$ picks up any bar lurking within a galaxy image. The time averaged pattern speed is calculated as the average rate of change of phase of the bar between the maximum and minimum time slices used. The pattern speed $\Omega_{\mathrm{p}}$ and the bar length $R_{\min }$ are used to calculate the rotation ratio, $\mathcal{R}=R_{\mathrm{co}} / R_{\mathrm{min}}$, where $R_{\mathrm{co}}$ is the corotation radius. All of the models for which this has been calculated fall into the generally accepted region for fast bars of $\mathcal{R}=1.2 \pm 0.2$ except for $\mathcal{R}_{16}=1.5$. However, there are large errors on the calculations of $\mathcal{R}$, mostly due to the uncertainty in the derivation of $R_{\min }$.

\section{Simulation results}

Sixteen simulations have been run and their positions within the parameter space defined by $1.12<Q_{\min }<1.26$ and $0.3<$ $f_{\mathrm{d}}<0.5$ are shown in Fig. 7. Outside of this parameter space it was not possible to generate two armed stellar spirals. The dashed line in Fig. 7 shows the additional constraint imposed by demanding that the softening length $s$, measured in cell lengths, satisfy $0.5<s<2.0$. Other parameters have a changeable canonical value, listed in Table 1. Data describing the initial conditions and results of each run are included in Appendix A. 


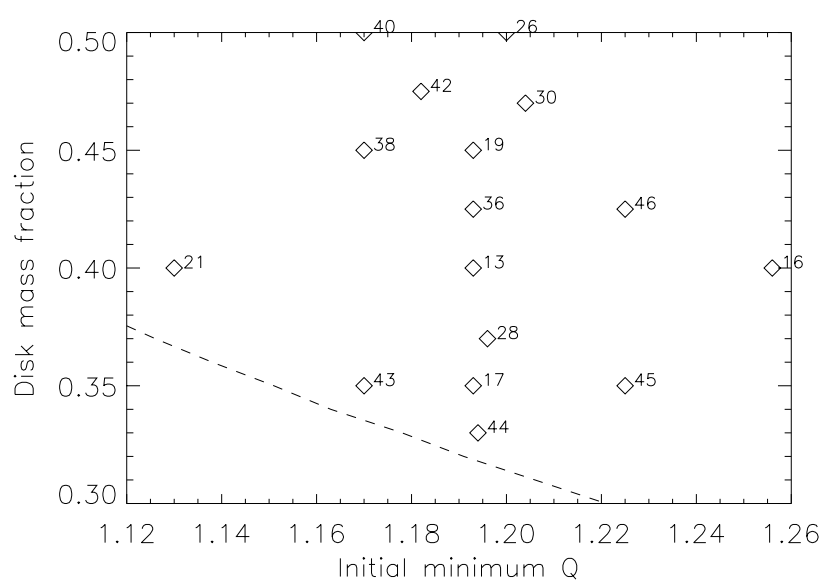

Fig. 7. Initial conditions for all. The dashed line marks the line of softening length $s=0.5$ grid cells.

Table 1. Canonical parameters.

\begin{tabular}{lll}
\hline \hline Number of particles & $N$ & $2 \times 10^{5}$ \\
Grid size & $I \times I$ & $250 \times 250$ \\
Simulation run time & $t_{\text {end }}$ & 160 \\
Time between data dumps & $t_{\text {dump }}$ & 2 \\
Courant number & $C$ & 0.75 \\
$N$-body time coefficient & $\varphi$ & 0.6 \\
Galaxy radius & $R_{\mathrm{G}}$ & 7.5 \\
Sound speed & $c$ & 0.025 \\
Gas mass & $M_{\mathrm{g}}$ & 0.01 \\
Cut off radius for stellar particles & $R_{\mathrm{cut}}$ & 5.0 \\
Rotation curve slope at $4 R_{\mathrm{d}}$ & $g_{4}$ & 0.0 \\
Gas recycling coefficient & $\alpha$ & 0.0 \\
\hline
\end{tabular}

$\dagger$ Cowie (1980).

\subsection{Computational checks}

Two checks ensure that each simulation behaves reasonably sensibly. The number of particles lost during the simulation $N_{\text {lost }}$ is always less than $0.5 \%$ of the total (1000) in all models and the position of the centre of mass of the stellar particles with respect to the grid centre drifts by less than $0.18 R_{\mathrm{d}}$. In five runs that no particles left the grid, total angular momentum was conserved to better than $0.002 \%$, and the ratio of kinetic energy to the virial of Clausius ${ }^{3}$ was unchanged from its initial value of 0.5 to better than $1 \%$.

${ }^{3}$ For a system of $i$ particles of mass $m$ at positions $\boldsymbol{r}_{i}$ and potential $\phi_{i}$, the virial of Clausius is $m \sum_{i} \boldsymbol{r}_{i} \cdot \nabla \phi_{i}$.

\subsection{Time evolution of simulations}

Figure 8 shows representative images and spectra at increasing times for sta19 and gas19. After a couple of bar rotation periods, the stars settle into a two armed spiral pattern. A similar pattern can be seen in the gas spectra, in addition to strong $m=3$ and $m=5$ patterns left over from the initial formation process. The stellar pattern does not change much over the next couple of rotation periods, while the higher order gaseous harmonics gradually disappear to leave a grand design spiral in the stars and the gas. At later times, the stars become dynamically hotter causing the spirals to fade somewhat and resulting in a more flocculent stellar distribution. However, the gas has a stronger memory than the stars and retains its grand design nature for longer.

This overall scenario is repeated in most simulations. However, the details vary, in particular how long it takes for the grand design stellar spirals to form and how soon afterwards the gas spirals lose their initial flocculence. There are also differences in how far the gas moves along the path between flocculence and grand design structure. In some simulations, multi armed gaseous structures can be observed at points along this path.

\subsection{Do these simulations reproduce the observations?}

i. Stellar and gaseous images can be decoupled. Every simulation has differing gaseous and stellar spectra, some significantly so, and these morphological differences are borne out in simulation images.

ii. Gaseous pitch angles are either similar to or tighter than stellar images. For simulations in which the gaseous images are two armed, the time averaged gaseous pitch angles are almost always smaller than those of the stellar images as illustrated in Fig. 9. This is in contrast to Block \& Puerari (1999) who find examples of Sc galaxies with tight $\left(\psi<20^{\circ}\right) K^{\prime}$ band spiral-arms. They do not, however, quote $B$ band pitch angles which makes comparison difficult, and their calulated angles depend on both inherent dynamics and dust contamination in the images.

iii. Stellar images are always grand design, whereas gas images can be flocculent. For almost every case in Fig. 9 the stellar image has a lower value of the flocculence $\mathcal{F}$ than the gaseous image. No stellar image has $\mathcal{F}>0.63$ whereas the gas images have $\mathcal{F}$ up to 0.68 or 1.4 times as flocculent in the sense of Sect. 4.3. This is borne out in the images, in which almost all of the stellar models have an obvious two armed grand design structure. Although few of the gaseous models are flocculent in the sense of Elmegreen \& Elmegreen (1982) i.e. many small scale arms, and many models possess an underlying two armed spiral, they also exhibit extra structures that are not seen in the relevant stellar model. These extra structures take the form of fronds leaving the main spiral or bridges between spiral-arms. Sometimes, extra partial spiral-arms can 

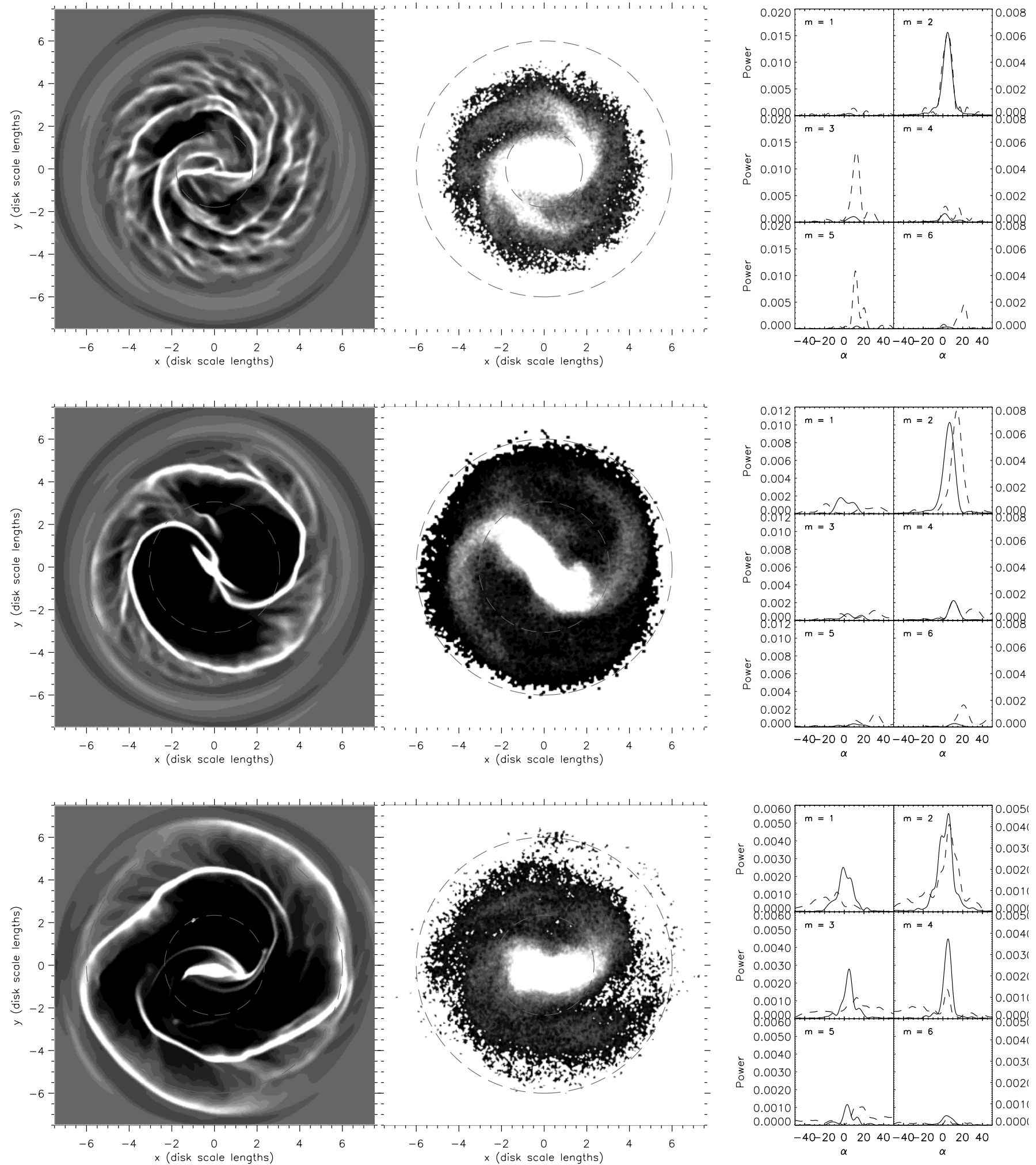

Fig. 8. Density fields and Fourier spectra for sta19 and gas 19 at 44, 80 and 110 time units or $2.0,3.7$ and 5.1 bar rotation periods. In the Fourier spectra, solid lines indicate the stars and dashed lines the gas.

also be seen. And in some instances, grand design stellar models do produce gaseous images that are truly multi armed or flocculent. These cases are rare but do arise. iv. Stellar images are one or two armed, gaseous images can be multi armed. This is seen to be true when looking at the images, and it is also apparent when comparing the power 

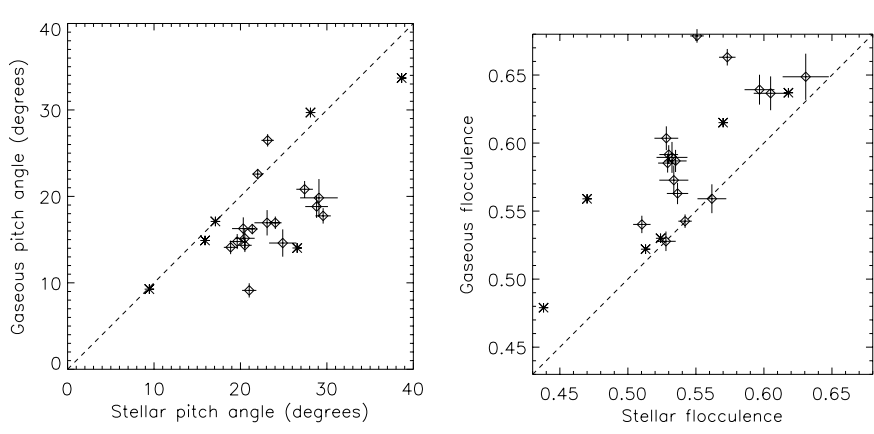

Fig. 9. The time averaged gaseous and stellar pitch angle $\psi$ and the flocculence $\mathcal{F}$ for all simulations. The dashed line is the line of equal pitch angle or flocculence. Error bars show parameters' values if $R_{\min }$ was changed by one increment. Asterisks indicate real galaxy images.

of the strongest component from each $m$ spectrum during every time slice. Almost all of the stellar spectra are dominated by the strongest $m=2$ component - the strongest component is $m \neq 2$ in only 5 out of the 311 time slices analyzed. Many gas spectra are also $m=2$ dominated but it is less common and the degree of dominance is generally much less - there are 32 gaseous time slices in which the strongest component is $m \neq 2$.

However, the only model which can truly be called multi armed is gas 16. For the majority of the time in which the stellar image exhibits a two armed spiral, the gas spectrum is dominated by an $m=4$ component. It has a similar morphology to our Galaxy as deduced from $K$ band and $240 \mu \mathrm{m}$ observations by Drimmel \& Spergel (2001) i.e. a two armed stellar spiral and a four armed gaseous spiral. SPH simulations of Englmaier \& Gerhard (1999) and Fux (1999) have also reproduced multiple arm gaseous spirals for our Galaxy.

v. Decoupling correlates with arm-interarm contrast. If the gaseous flocculence is considered, there is a significant correlation with the arm-interarm contrast, (see Fig. 10, correlation coefficient -0.77$)$. Since the observations and the simulations show that $K^{\prime}$ band and stellar images are generally not flocculent, there is a good case for using the flocculence of the gas, and hence the arm-interarm contrast, as a guide to the amount of decoupling.

It is reasonable to assume that the stellar arm-interarm contrast is not only an indicator of decoupling between stellar and gaseous morphologies but also its cause. This is particularly true of the non self gravitating case, where the driver of morphological structure within the gas is the non-axisymmetric part of the stellar potential. Hence when the arm-interam contrast is low, the noise in the stellar component makes a significant contribution to the driving force, leading to many low amplitude features in the gas and an overall flocculent structure. But when the contrast is high, the stellar spiral dominates, creating a single high amplitude wave in the gas and a grand design spiral. This can be seen in the gaseous arm-interarm contrasts: $C_{16}=3.08$ for the flocculent simulation and $C_{19}=6.13$ for the grand design simulation.
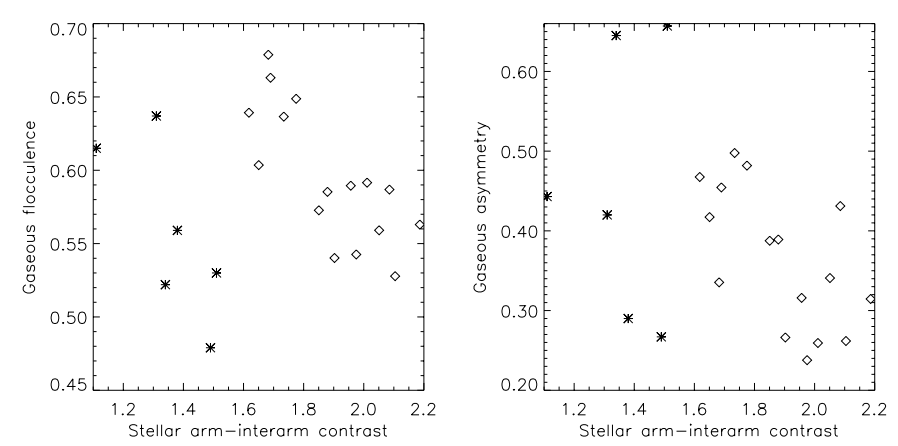

Fig. 10. Correlation of time averaged arm-interarm contrast $C$ with gaseous flocculence $\mathcal{F}_{\text {gas }}$ (left panel) and gaseous asymmetry $\mathcal{A}_{\text {gas }}$ (right panel). Asterisks indicate values for real galaxy images.
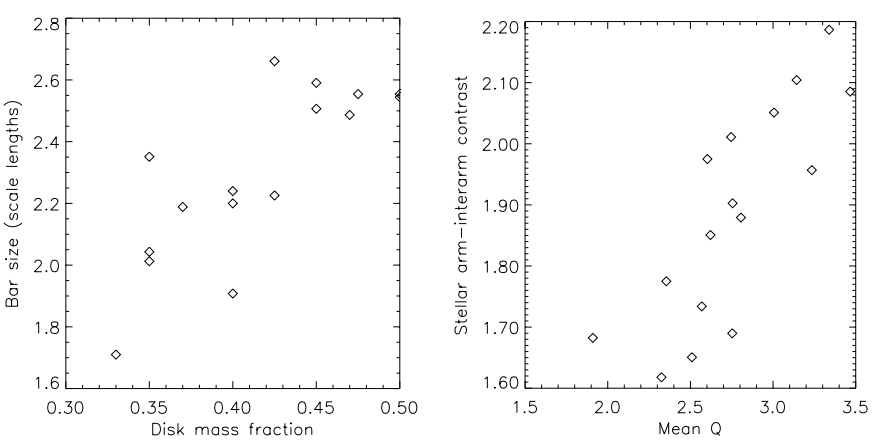

Fig. 11. Left panel: correlation of disk mass fraction at four scale lengths $f_{\mathrm{d}}$ with the time averaged bar size as defined by the parameter $R_{\min }$. Right panel: correlation of the time averaged mean value of $Q$ with time averaged stellar arm-interarm contrast $C$.

This relationship has also been noticed in real galaxies. Four galaxies that are obviously flocculent in the optical were observed by Thornley (1996) in the infrared. She found good evidence for low amplitude grand design structures with $K^{\prime}$ band arm-interarm contrasts of just 1.1 to 1.4. In real galaxies, however, a combination of supernovae and differential rotation can give rise to self-propagating star formation, causing flocculence in optical images irrespective of the underlying potential (Mueller \& Arnett 1976; Gerola \& Seiden 1978).

\subsection{Can these models predict galactic structure parameters?}

The modal theory of spiral structure predicts that galaxy morphology should be directly related to the underlying structure of the galaxy (Bertin et al. 1989). I now examine whether this is true in my models.

i. Galaxy disk mass correlates with bar size. Figure 11 shows the strong relationship between the disk mass fraction at four scale lengths $f_{\mathrm{d}}$ and the size of the bar as defined by the parameter $R_{\min }$. The correlation coefficient is 0.80 . 
ii. Stellar $Q$ correlates with flocculence. The gaseous flocculence and $\bar{Q}(t)$ and correlate with a coefficient of -0.62 . This is in the opposite direction to the correlation predicted by the modal theory in Elmegreen \& Thomasson (1993) who state that flocculent spiral structure arises when $Q>2.5$. This is probably due to the fact that, unlike Elmegreen \& Thomasson (1993), DUAL has no stellar cooling algorithm. It would be interesting to compare my results with S0 galaxies, since they have very little gas and hence little chance to cool their stars dynamically.

$\bar{Q}(t)$ correlates strongly with the time averaged stellar arminterarm contrast (coefficient of 0.81) as shown in Fig. 11. This probably results from the scattering of stellar particles from the spiral-arms, with higher contrast leading to greater scattering and dynamically hotter disks.

\subsection{Comparison with observations}

It is important to compare the simulations with observations of real galaxies to ensure that they are not just interesting computer simulations but that any conclusions drawn from them are relevant to the real universe.

The simulated time averaged arm-interarm contrasts are similar to those of real galaxies. From 18 spiral galaxies, Rix $\&$ Zaritsky (1995) find that $C$ has an average value of $\sim 2$, with large variations above and below this value. Rix (1993) finds that $1.8<C<3.0$ for the grand design galaxy M 51. The arminterarm contrast is found to be somewhat lower for the flocculent galaxies studied by Thornley (1996) who concludes that $1.1<C<1.4$. My models lie between $1.6<C<2.2$.

Data from Kennicutt (1981) shows that the gaseous stellar pitch angles in Sc galaxies occupy a similar range to that of the models, $10^{\circ}<\psi_{\text {gas }}<30^{\circ}$. And the rotation curves in Fig. 2 show that the mass distributions are similar to both disk and halo dominated Sc galaxies.

Many of the figures above include observations of six real galaxies in the $K^{\prime}$ and $B$ bands. The parameters associated with these observations have been derived using a similar procedure to that used on the models. The galaxies are NGC 2997, NGC 3223, NGC 5085, NGC 3810, NGC 3893 and NGC 5364. The data for the first three were kindly donated by David Block, and for the last three by Thilo Kranz. These data show similar relationships for pitch angle and flocculence (Fig. 9) to the models with $K^{\prime}$ band and $B$ band data in place of $N$-body and hydrodynamical results. The arm-interarm contrasts for the real galaxies show a correlation with flocculence similar to the models (Fig. 10) but with the arm-interarm contrasts offset by $\Delta C \sim 0.6$. The six galaxies are, however, correctly ranked in order of $B$ band flocculence.

\section{Discussion}

The simulations in this paper have reproduced many of the observed differences between $B$ and $K^{\prime}$ band images of galaxies. At first glance this seems slightly bizarre, since the motion of the gas should just follow that of the underlying stellar potential. But, due to the long range nature of gravity, the potential generally does not follow the density which generates it (as can be seen in Fig. 12).

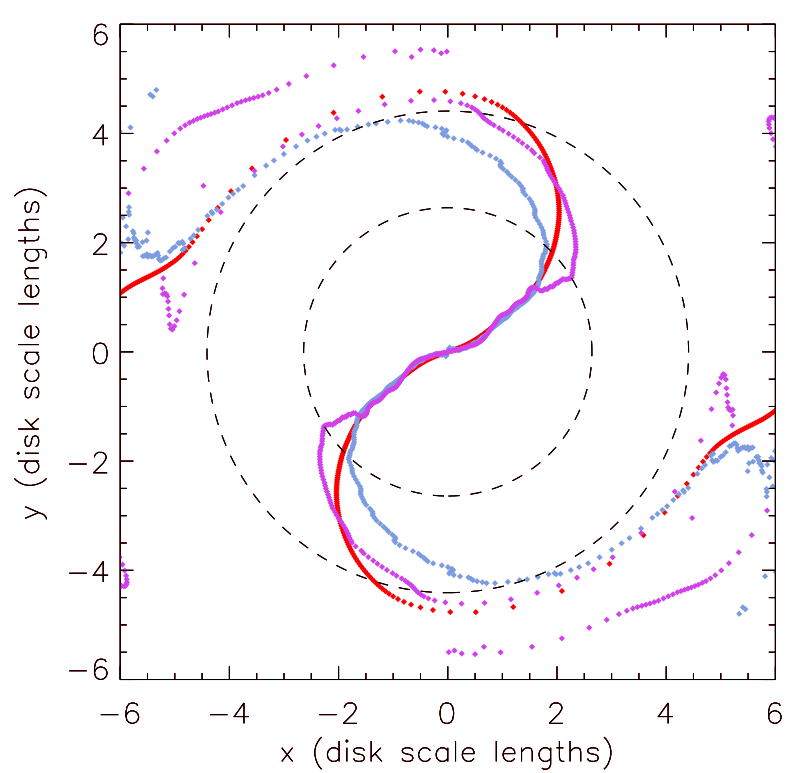

Fig. 12. Position of the $m=2$ maxima for grand design model. The light grey diamonds mark the stellar maxima, the dark grey diamonds mark the gaseous maxima and the black diamonds mark the minima of the stellar potential. Diamonds are equally spaced in radius. The dashed lines mark $R_{\min }=2.6$ and $R_{\mathrm{OLR}}=4.4$.

\subsection{The pitch angles of gaseous and stellar spirals}

In order to explain the secular evolution of spiral galaxies, Zhang (1996) presents a derivation of a phase shifted, self consistent, stellar density and potential pair. Figure 12, which shows the maxima of the $m=2$ Fourier components of the gas density, stellar density and stellar potential, indicates that this type of effect may be at work in my simulations. The gas follows the displaced stellar potential rather than the stellar density, particularly outside of the bar region. The gas maxima turn from the bar region into spirals much later than the stars, possibly helped by $x_{1}$ orbits inside the bar region, thereby providing an explanation for the lower gaseous pitch angles.

\subsection{The flocculence of gaseous and stellar spirals}

Much of the difference in the smaller scale structure between the gaseous and stellar images can be explained in terms of the dynamics of the two components. Structures in the stellar disk get strongly amplified in the dynamically cold gas, leading to shocks. Since the sound speed in the gas is much lower than the velocity dispersion of the stars, and since gas is collisional, gaseous structures are much sharper and longer lasting than stellar ones. Hence the history of the simulation is important as well as any particular snapshot in explaining differences in morphology. This would not have been apparent if an analytical potential was used in place of an evolving disk of particles for the stellar component of the models.

When a two armed density wave forms in the stellar disk, if it is fast and strong enough, it will wipe away the smaller amplitude features in the gas, particularly in the inner regions 
where it is strongest. The alternative is a low amplitude stellar spiral leading to flocculent gas images. This can be seen in the gas velocity fields where flocculent models have much weaker gas velocity divergences (and hence shocks), particularly outside corotation.

The systematically high values of stellar arm-interarm contrast seen in Fig. 10 may be the reason why truly flocculent models have been hard to find. Flocculent structures in real galaxies are thought to be due to coherent star forming regions (Mueller \& Arnett 1976; Gerola \& Seiden 1978). Hence, star formation algorithms may promote flocculent gas spirals in these models.

\subsection{Intrinsic characteristics of galaxies}

It has been shown that decoupling of stellar and gaseous morphologies is a function of stellar arm-interarm contrast. The arm-interarm contrast is an observable quantity which, using Fig. 11, yields a rough value for $\bar{Q}(t)$. Similarly, the bar length correlates with the disk mass.

Before these trends can be applied to real galaxies, it is worth considering how they would be affected by changes in the models. Many N-body simulations include techniques to keep the stellar particles dynamically cooled and hence prolong the life of the spiral structure. From the discussion in Sect. 5.2, it is likely that this would lead to more grand design spiral structure in the gas. A similar effect may happen if an ILR were included in the models, perhaps by adding a bulge component. A bulge may also reduce the strength of the bars and lower the artificially high stellar arm-interarm contrasts in the simulations. This would provide a good opportunity to search for unbarred or weakly barred models.

Since gas mass discriminates between galaxies along the Hubble sequence, it is essential that gaseous self gravity is added to the current model. Gas with a few per cent of the total mass would aid the stellar cooling process since the gas is dynamically cool. Weak gravitational coupling between the two components could lead to greater similarity in form. However, local gaseous features would be enhanced by gaseous self gravity, decoupling the morphology. Gas with too much self gravity (above $\sim 10 \%$ of the total mass) could become lumps, scattering the stars and heating the disk (Junqueira \& Combes 1996).

\section{Conclusions}

The DUAL code combines $N$-body and finite-difference hydrodynamic techniques to reproduce some of the differences in the morphology of $K^{\prime}$ and $B$ band spiral galaxies. It is assumed that $N$-body simulations mimic the dynamics of the underlying stellar population seen in the $K^{\prime}$ band and that morphologies produced by hydrodynamic modelling can be directly compared to $B$ band images. Given this, the simulations have verified the principle of morphological decoupling and many of the detailed results of $K^{\prime}$ and $B$ band galaxy observations.

At present there are no statistics for how prevalent decoupling is in real galaxies. Hence, the extremes of the observations mentioned above should be viewed as instances of a common, but possibly not dominant, phenomenon. The lack of decoupling in many spiral galaxies may account for the difficulty in creating simulations with radically different morphologies in the stellar and gaseous components. It would be very helpful to analyze a statistically meaningful sample of galaxies in $B$ and $K^{\prime}$ bands to determine the distribution of decoupling in real galaxies, particularly the differences in flocculence and pitch angle.

I have introduced a numerical value to characterize the flocculence of a galaxy, $\mathcal{F}$, closely related to the uniformity of the Fourier spectra. It would be useful to calculate the value of $\mathcal{F}$ for many more real galaxies and compare the results to the arm classes of Elmegreen \& Elmegreen (1982).

Gaseous spirals are shown to have tighter pitch angles than stellar spirals. This was tentatively attributed to a phase shift between the stellar density and the potential, shown to exist in at least one of the models. Is this effect present in real galaxies with pitch angle differences? Do the simulations exhibit the characteristic accretion and excretion of stellar particles from the corotation radius?

I have shown that morphological decoupling increases as the stellar arm-interarm contrast falls. Hence flocculent galaxies contain low amplitude stellar arms. High arm-interarm contrasts lead to a hotter disk, so it is possible to make predictions about $Q$ from the strength of the arms. The second parameter controlling the models, the disk mass, can be predicted from the size of the stellar bar. However, the $N$-body simulations proceed in a very non-linear fashion, so it is not possible to draw any conclusions about the initial value of $Q$ from the end state of the run.

To determine the relative importance of star formation, dust and the underlying dynamics in defining the morphology of a galaxy, a better understanding is needed of how simulations relate to $B$ band images, or alternatively $\mathrm{H} \mathrm{I}$ or $\mathrm{CO}$ data. It would be helpful to understand how morphological parameters, particularly the pitch angle and the flocculence, respond to the addition of dusty structures and star forming knots to the simulated images.

The use of $\mathrm{N}$-body techniques, instead of an analytic potential, proved to be essential in reproducing the dual nature of stellar and gaseous morphologies. However, the self gravity of the gas and star and dust formation algorithms must be added to make the DUAL code fully self consistent and capable of modelling true spiral galaxies.

It was expected that not all of the morphological complexity and variety of spiral galaxies in the $B$ band would be explained by purely dynamical models. However, the similarities between these simulations and the observations imply that much of the dual morphologies in spiral galaxies is due to the difference in the dynamics of stars and gas.

Acknowledgements. I thank James Binney for helpful discussions and advice. Thanks also to Jerry Sellwood for providing me with the $N$-body code which, in an amended form, is half of the DUAL code. PPARC generously provided much of the funding for my research. 


\section{Appendix A: Dual morphology models}

Table A.1. Results for every simulation: the disk mass fraction at four scale lengths $f_{\mathrm{d}}$, the minimum value of the initial $Q$ parameter $Q_{\text {min }}$, the effective scale height of the stellar particles $z_{0}$, the time averaged length of the bar used as the minimum radius in Fourier decompositions $R_{\text {min }}$, the rotation ratio $\mathcal{R}$, the time averaged pitch angle of the stellar spirals $\psi_{\text {sta }}$, the time averaged pitch angle of the gaseous spirals $\psi_{\text {gas }}$, the time averaged stellar flocculence $\mathcal{F}_{\text {sta }}$, the time averaged gaseous flocculence $\mathcal{F}_{\text {gas }}$ and the time averaged stellar arm-interarm contrast $C$.

\begin{tabular}{lllllllllll}
\hline \hline Run & $f_{\mathrm{d}}$ & $Q_{\min }$ & $z_{0}$ & $R_{\min }$ & $\mathcal{R}$ & $\psi_{\text {sta }}$ & $\psi_{\text {gas }}$ & $\mathcal{F}_{\text {sta }}$ & $\mathcal{F}_{\text {gas }}$ & $C$ \\
\hline 13 & 0.40 & 1.193 & 0.120 & 2.31 & 1.01 & 21.0 & 9.1 & 0.57 & 0.66 & 1.70 \\
16 & 0.40 & 1.256 & 0.133 & 1.99 & 1.49 & 23.1 & 26.5 & 0.55 & 0.68 & 1.68 \\
17 & 0.35 & 1.193 & 0.108 & 2.04 & 1.09 & 24.9 & 14.6 & 0.60 & 0.64 & 1.73 \\
19 & 0.45 & 1.193 & 0.131 & 2.58 & 0.98 & 21.4 & 16.2 & 0.53 & 0.53 & 21.0 \\
21 & 0.40 & 1.130 & 0.108 & 2.28 & 1.04 & 22.0 & 22.6 & 0.54 & 0.54 & 1.97 \\
26 & 0.50 & 1.200 & 0.144 & 2.54 & 1.11 & 28.8 & 18.8 & 0.54 & 0.56 & 2.19 \\
28 & 0.37 & 1.196 & 0.114 & 2.19 & 1.08 & 20.3 & 16.2 & 0.53 & 0.57 & 1.85 \\
30 & 0.47 & 1.204 & 0.138 & 2.49 & 1.02 & 29.6 & 17.7 & 0.56 & 0.56 & 2.05 \\
36 & 0.43 & 1.193 & 0.126 & 2.23 & 1.07 & 20.5 & 15.1 & 0.53 & 0.60 & 1.65 \\
38 & 0.45 & 1.170 & 0.126 & 2.23 & 1.07 & 20.5 & 14.3 & 0.51 & 0.54 & 1.90 \\
40 & 0.50 & 1.170 & 0.137 & 2.55 & 1.02 & 24.0 & 16.9 & 0.53 & 0.59 & 1.96 \\
42 & 0.48 & 1.182 & 0.134 & 2.46 & 1.06 & 27.4 & 20.8 & 0.54 & 0.59 & 2.09 \\
43 & 0.35 & 1.170 & 0.104 & 2.35 & 0.98 & 19.6 & 14.8 & 0.53 & 0.59 & 2.01 \\
44 & 0.33 & 1.194 & 0.104 & 1.70 & 1.24 & 29.1 & 19.8 & 0.63 & 0.65 & 1.78 \\
45 & 0.35 & 1.225 & 0.114 & 1.99 & 1.04 & 23.1 & 16.9 & 0.60 & 0.64 & 1.62 \\
46 & 0.43 & 1.225 & 0.133 & 2.63 & 0.99 & 18.9 & 14.1 & 0.53 & 0.59 & 1.88 \\
\hline
\end{tabular}

Table A.2. Results for six real galaxies: the length of the bar used as the minimum radius in Fourier decompositions $R_{\min }$, the pitch angle of the stellar spirals $\psi_{\text {sta }}$, the pitch angle of the gaseous spirals $\psi_{\text {gas }}$, the stellar flocculence $\mathcal{F}_{\text {sta }}$, the gaseous flocculence $\mathcal{F}_{\text {gas }}$ and the stellar arm-interarm contrast $C$.

\begin{tabular}{lllllll}
\hline \hline NGC & 2997 & 3223 & 3810 & 3893 & 5085 & 5364 \\
\hline$R_{\min }$ & $34^{\prime \prime}$ & $15^{\prime \prime}$ & $8.3^{\prime \prime}$ & $10^{\prime \prime}$ & $25^{\prime \prime}$ & $29^{\prime \prime}$ \\
$R_{\text {max }}$ & $122^{\prime \prime}$ & $48^{\prime \prime}$ & $116^{\prime \prime}$ & $123^{\prime \prime}$ & $50^{\prime \prime}$ & $162^{\prime \prime}$ \\
$\psi_{\text {sta }}$ & 26.6 & 9.46 & 17.1 & 15.9 & 38.7 & 28.1 \\
$\psi_{\text {gas }}$ & 14.0 & 9.27 & 17.1 & 14.9 & 33.7 & 29.7 \\
$\mathcal{F}_{\text {sta }}$ & 0.44 & 0.57 & 0.51 & 0.52 & 0.47 & 0.62 \\
$\mathcal{F}_{\text {gas }}$ & 0.48 & 0.62 & 0.52 & 0.53 & 0.56 & 0.64 \\
$C$ & 1.49 & 1.11 & 1.34 & 1.51 & 1.38 & 1.31 \\
\hline
\end{tabular}

\section{References}

Berman, S. 2001, A\&A, 371, 476

Bertin, G., \& Lin, C. C. 1996, Spiral structure in galaxies (Cambridge: MIT Press)

Bertin, G., Lin, C. C., Lowe, S. A., \& Thurstans, R. P. 1989, ApJ, 338, 78

Binney, J., \& Merrifield, M. 1998, Galactic Astronomy (Princeton: Princeton University Press)

Block, D. L., \& Puerari, I. 1999, A\&A, 342, 627

Cowie, L. L. 1980, ApJ, 236, 868

Danver, C. G. 1942, Ann. Obs. Lund, 10, 7

de Grijs, R., \& Peletier, R. F. 1997, A\&A, 320, L21

Dehnen, W. 1999, AJ, 118, 1201

Dehnen, W., \& Binney, J. J. 1998, MNRAS, 298, 387
Drimmel, R., \& Spergel, D. N. 2001, ApJ, 556, 181

Elmegreen, B. G., \& Thomasson, M. 1993, A\&A, 272, 37

Elmegreen, D. M., \& Elmegreen, B. G. 1982, MNRAS, 201, 1021

Englmaier, P., \& Gerhard, O. 1997, MNRAS, 287, 57

Englmaier, P., \& Gerhard, O. 1999, MNRAS, 304, 512

Eskridge, P., Frogel, J., Pogge, R., et al. 2000, AJ, 119, 536

Fux, R. 1999, A\&A, 345, 787

Gerola, H., \& Seiden, P. E. 1978, ApJ, 223, 129

Grosbol, P. J., \& Patsis, P. A. 1998, A\&A, 336, 840

Iye, M., Okamura, S., Hamabe, M., \& Watanabe, M. 1982, ApJ, 256, 103

Jaynes, E. T. 1958, Probability theory in science and engineering, Colloquium Lectures in Pure and Applied Science, 4, Socony-Mobil Oil Co., Dallas

Junqueira, S., \& Combes, F. 1996, A\&A, 312, 703

Kennicutt, R. C. 1981, AJ, 86, 1847

Mueller, M. W., \& Arnett, W. D. 1976, ApJ, 210, 670

Rhoads, J. E. 1998, AJ, 115, 472

Rix, H. 1993, PASP, 105, 999

Rix, H., \& Zaritsky, D. 1995, ApJ, 447, 82

Saraiva Schroeder, M. F., Pastoriza, M. G., Kepler, S. O., \& Puerari, I. 1994, A\&AS, 108, 41

Swaters, R. A., Madore, B. F., \& Trewhella, M. 2000, ApJ, 531, L107

Thornley, M. D. 1996, ApJ, 469, L45

Toomre, A. 1964, ApJ, 139, 1217

van Albada, G. D., \& Roberts, W. W. 1981, ApJ, 246, 740

van Albada, G. D., van Leer, B., \& Roberts, W. W. 1982, A\&A, 108, 76

van der Kruit, P. C., \& Searle, L. 1981, A\&A, 95, 105

Zhang, X. 1996, ApJ, 457, 125 